\title{
Development and Validation of a Nomogram for Predicting Obstructive Sleep Apnea in Bariatric Surgery Candidates
}

\author{
Wenhui Chen ${ }^{1}$ * \\ Jia Feng ${ }^{2, *}$ \\ Yucheng Wang' \\ Cunchuan Wang' \\ Zhiyong Dong'
}

\section{On behalf of Chinese \\ Obesity and Metabolic Surgery Collaborative}

'Department of Metabolic and Bariatric Surgery, The First Affiliated Hospital of Jinan University, Guangzhou, People's Republic of China; ${ }^{2}$ Department of Cellular Biology, Institute of Biomedicine, Jinan University, Guangzhou, People's Republic of China

*These authors contributed equally to this work
Correspondence: Zhiyong Dong;

Cunchuan Wang

Department of Metabolic and Bariatric Surgery, The First Affiliated Hospital of Jinan University, No. 613 Huangpu Avenue West, Guangzhou, Guangdong,

510632, People's Republic of China

Tel +86 I35 44527726

Fax +86 2038688608

Email dongzy2008@jnu.edu.cn;

twcc2015@163.com
Background: Obstructive sleep apnea (OSA) is highly prevalent in bariatric surgery patients and can lead to potential perioperative risks, but some screening tools lack adequate performance in this population. Thus, we aimed to develop and validate a clinical nomogram for predicting OSA in bariatric surgery candidates.

Methods: We retrospectively collected the data of 482 bariatric surgery patients between September 2015 and January 2020. Patients were randomly classified into training cohort $(n=338)$ and validation cohort $(n=144)$. The Lasso regression was used to select potentially relevant features; then, multivariable logistic regression analysis was used to establish the nomogram. Discrimination, calibration and clinical usefulness of the nomogram were assessed using the $\mathrm{C}$-index, calibration curve and decision curve analysis (DCA).

Results: The overall prevalence of OSA was $71.0 \%$ and higher in males (88.2\%) compared to females $(60.1 \%)$. Of these, $26.1 \%$ had mild OSA, $14.9 \%$ had moderate OSA, and $44.8 \%$ had severe OSA. The nomogram consisted of gender, habitual snoring, type 2 diabetes mellitus (T2DM), neck circumference, body mass index (BMI) and age. The nomogram provided favorable discrimination, with a $\mathrm{C}$-indexes for the training and validation cohort of 0.856 (95\% CI: 0.816-0.897) and 0.829 (95\% CI: 0.76-0.895), respectively, and good calibration. The DCA displayed that the nomogram was clinically useful.

Conclusion: We established a concise and practical nomogram that could facilitate the preoperative individualized prediction of OSA before bariatric surgery, which may help clinicians select bariatric surgery patients with high-risk OSA for polysomnography (PSG).

Keywords: obstructive sleep apnea, bariatric surgery, obesity, predictors, nomogram

\section{Introduction}

Obstructive sleep apnea (OSA) is a common sleep-related disorder that is characterized by repeated episodes of partial or complete upper airway obstruction during sleep, resulting in repetitive hypoxemia, sleep fragmentation, morning headaches and excessive daytime sleepiness. ${ }^{1-3}$ Long-term complications of OSA include type 2 diabetes mellitus (T2DM), hypertension, cardiovascular disease (atrial fibrillation, heart failure and coronary heart disease), stroke, and sudden-death during sleep. ${ }^{1,4,5}$ Among the multifactorial etiologies for OSA development, obesity is a well-known risk factor and predictor, and approximately $70 \%$ of patients with diagnosed OSA are obese. ${ }^{6-8}$

Recently, bariatric surgery has been increasing in popularity because it achieves sustained weight loss and improves obesity-related complications. ${ }^{9}$ In patients evaluated for bariatric surgery, the prevalence of OSA ranges from $59.9 \%$ to $93.5 \%$ 
depending on the demographic characteristics of the participants. ${ }^{10}$ Although the prevalence of OSA is high in bariatric surgery candidates, its underdiagnosis is a common issue. A prospective study of 834 patients showed that approximately $40 \%$ of bariatric surgery candidates had no previous diagnosis or had not undergone any screening before starting preoperative evaluation. ${ }^{11}$ Bariatric surgery patients with OSA have greater anesthetic risk, higher risk for perioperative and postoperative complications. ${ }^{12,13}$ The underdiagnosis OSA may lead to devastating complications and potentially increase a patient's morbidity and mortality, suggesting the early diagnosis and management of OSA is extremely important. Thus, the routine clinical screening for OSA should be recommended in the bariatric population, although some patients with OSA may be asymptomatic. ${ }^{14,15}$

Currently, nocturnal polysomnography (PSG) remains the gold standard for diagnosing OSA but it is expensive, time-consuming, and uncomfortable for patients, which makes some patients refuse to undergo PSG. Therefore, this diagnostic tool is difficult to be widely available in patients with suspected OSA, especially in economically underdeveloped areas. Although several questionnaires (Berlin Questionnaire, STOP-Bang, GOAL and Fatigue Severity Scale (FSS)) are available for detecting patients with high risk of OSA, ${ }^{16,17}$ their application value in patients who undergo bariatric surgery remains unclear, and further validation is warranted. A prospective study of 411 bariatric surgery patients showed No-Apnea, STOPBang questionnaire and NoSAS score were useful tools for screening of OSA in morbidly obese patients, but their accuracy was less than $80 \% .{ }^{18}$ In another study with 425 bariatric surgery patients, Epworth Sleepiness Scale (ESS) was considered to have no significant predictive value before bariatric surgery. ${ }^{19}$ In addition, some study showed there were gender-specific differences in the performance of screening questionnaires (ESS, FSS, STOP-Bang, and NoSAS) in the bariatric population. ${ }^{20}$ Duarte et al developed a tool of NO-OSAS to identify moderate to severe OSA among bariatric surgery patients, but its performance needs to be further verified in other cohorts. ${ }^{21}$ Therefore, we need to further explore better screening tools to screen for OSA in the bariatric population.

Nomogram is a concise and practical tool for predicting risk by incorporating important predictors to predict clinical events and outcomes, ${ }^{22,23}$ and it has been widely used to predict the risk and prognosis of various diseases. The present study aimed to develop and validate a clinical nomogram for accurately predicting the risk of OSA based on preoperative clinical features in bariatric surgery candidates.

\section{Methods}

\section{Patient Population}

The data of consecutive patients who planned to undergo bariatric surgery were retrospectively collected in our hospital between September 2015 and January 2020. Inclusion criteria included the following: patients aged 18 to 65 years old, $B M I \geq 35 \mathrm{~kg} / \mathrm{m}^{2}$ or $\mathrm{BMI} \geq 27.5 \mathrm{~kg} / \mathrm{m}^{2}$ combined with inadequately controlled T2DM and at least one other metabolic disease ${ }^{24}$ patients agreed to be screened preoperatively by polysomnography. Exclusion criteria were as follows: alcohol or drug abuse, severe depression, other severe diseases contraindicating bariatric surgery, missing or incomplete data, recording failed or recording time less than 300 min. The study was approved by the ethics committee of the First Affiliated Hospital of Jinan University. Furthermore, it was performed in accordance with the principles of the Declaration of Helsinki. Written informed consent from all eligible patients was obtained before bariatric surgery.

\section{Data Collection}

Demographic data were collected, including gender, age, weight and height, as well as neck circumference, chest circumference, waist circumference and hip circumference. We evaluated waist-to-hip ratio (WHR), waist-toheight ratio (WtHR) and neck-to-height ratio (NHtR) based on these parameters. In addition, we also collected the data on co-morbidity (fatty liver, hypertension, T2DM, hyperlipidemia, hyperuricemia). Neck circumference was measured at the level of the laryngeal prominence; chest circumference was measured at the level of nipple point; waist circumference was measured at the level of the umbilicus; hip circumference was measured at the horizontal circumference of the most prominent part of the hips backwards. BMI was calculated using a formula that divides weight by height into squares. Habitual snoring was defined as a snoring frequency $\geq 3$ nights per week. ${ }^{25}$ Fatty liver was diagnosed with color ultrasound. Criteria for the diagnosis of Hypertension were systolic blood pressure $\geq 140 \mathrm{~mm} \mathrm{Hg}$ or diastolic blood pressure $\geq 90 \mathrm{~mm}$ $\mathrm{Hg}$, a history of hypertension or the use of antihypertensive drugs. Criteria for the diagnosis of T2DM was fasting plasma glucose $(\mathrm{FPG}) \geq 7.0 \mathrm{mmol} / \mathrm{L}(126 \mathrm{mg} / \mathrm{dL})$, random plasma glucose $\geq 11.1 \mathrm{mmol} / \mathrm{L}(200 \mathrm{mg} / \mathrm{dL})$, 2-hour plasma 
glucose value after a 75 -g oral glucose tolerance test $($ OGTT) $\geq 11.1 \mathrm{mmol} / \mathrm{L}(200 \mathrm{mg} / \mathrm{dL})$, a history of T2DM or the use of antihyperglycemic drugs. Criteria for the diagnosis of Hyperlipidemia were serum triglycerides above $1.70 \mathrm{mmol} / \mathrm{L}$, serum total cholesterol above $5.7 \mathrm{mmol} / \mathrm{L}$, serum low-density lipoprotein (LDL) cholesterol above $3.76 \mathrm{mmol} / \mathrm{L}$, serum high-density lipoprotein (HDL) cholesterol less than $0.91 \mathrm{mmol} / \mathrm{L}$, or the use of lipid-lowering drugs. Hyperuricemia was defined as serum uric acid above $420 \mathrm{mmol} / \mathrm{L}$ in men and above $360 \mathrm{mmol} /$ $\mathrm{L}$ in women.

\section{Sleep Study}

All included patients underwent full-night PSG (Compumedics E-series, Australia), consisting of oronasal airflow (using dual pressure and thermal sensors), respiratory effort (abdomen and thorax), electrocardiogram, electrooculogram, electroencephalogram, snoring using a tracheal microphone, body position and oxygen saturation (using Nonin finger probe). An experienced sleep specialist scored manually and interpreted polysomnographic recordings in accordance with established guidelines. ${ }^{26}$ The data of sleep variables were collected from the PSG as follows: total sleep time, sleep efficiency, apnea-hypopnea index (AHI), arousal index, mean oxygen saturation and minimum oxygen saturation.

Apnea was defined as a complete cessation of oronasal airflow for at least 10 seconds. ${ }^{26}$ Hypopneas were defined as a reduction $\geq 30 \%$ of pre-event baseline in the respiratory signal, for $\geq 10$ seconds in association with either $\geq 3 \%$ arterial oxygen desaturation or an arousal. ${ }^{22}$ Apnea-hypopnea index (AHI) was defined as the number of apnea and hypopnea events per hour. ${ }^{26}$ OSA severity was classified according to AHI thresholds: AHI less than 5 events/h was considered normal, AHI 5-15 events/h was considered as mild OSA, AHI 15-30 events/h as moderate OSA and AHI more than 30 events/h as severe OSA. ${ }^{26}$

\section{Statistical Analysis}

Statistical analysis was performed using SPSS 13.0 for Windows (SPSS Inc. Chicago, IL, USA), R software (version 4.0.2 http://www.Rproject.org) and MedCalc version 19.4.0 (Ostend, Belgium). Normally distributed continuous variables are represented by the mean \pm standard deviation (SD); non-normal continuous variables are expressed as the median (interquartile range); categorical variables are reported as the number (percentage). Comparison between two groups were examined using Student's $t$-test or Mann-
Whitney test for continuous data, and Chi-square test or Fisher's exact test for categorical data.

A permuted randomization number table generated by using the "caret" package of R was applied to randomly assign the patients to the training cohort and validation cohort in a 7:3 ratio. The least absolute shrinkage and selection operator (LASSO) method is used to reduce the dimensionality of data, ${ }^{27}$ and the optimal predictive features from the training cohort were identified. Features with nonzero coefficients in the LASSO regression model were selected. Then, the features selected in the LASSO regression model were included in the multivariate logistic regression to develop a nomogram. Calibration curves were plotted to assess the calibration of this model, accompanied by the Hosmer-Lemeshow test ${ }^{27}(\mathrm{P}>0.05$ is considered as the goodness of calibration); an ideal calibration curve would perfectly fit the 45-degree reference line. The concordance index (C-index) was used to evaluate the discrimination of the nomogram. The nomogram was subjected to bootstrapping validation (1000 bootstrap resamples) to calculate a relatively corrected $\mathrm{C}$-index. Decision curve analysis (DCA) was performed to evaluate the clinical usefulness of the nomogram based on net benefits at different threshold probabilities in the training and validation cohort. ${ }^{28}$ Area under the curve (AUC) in receiver operating characteristic (ROC) analysis was used to evaluate predictive accuracy. $\mathrm{P}$ value of less than 0.05 was considered statistically significance.

\section{Results}

\section{Basic Characteristics of Patients}

A total of 499 patients who underwent full-night PSG screened for inclusion, 17 patients were subsequently excluded: because of missing or incomplete data, recording failed, recording time less than $300 \mathrm{~min}$. Finally, 482 patients were enrolled to the study, 296 (61.4\%) women and $186(38.6 \%)$ men. Of those 482 patients, 342 (71.0\%) patients fulfilled the diagnostic criteria for OSA, specifically $126(26.1 \%)$ with mild OSA, 72 (14.9\%) with moderate OSA and $144(44.8 \%)$ with severe OSA. In male patients, the prevalence was $88.2 \%$ (164/186), compared to $60.1 \%(178 / 296)$ in female subjects $\left(\mathrm{x}^{2}=43.57, \mathrm{P}=0.000\right)$.

Four hundred and eighty-two patients were randomly assigned to either the training cohort $(n=338$, for development of the nomogram) or the validation cohorts $(n=144$, for validation of the nomogram). There is no significant difference in basic characteristics between the training 
cohort and the validation cohort (all $\mathrm{P}>0.05$ ), but compared with non-OSA group, the OSA group had a relatively higher proportion of males, larger demographic variables (weight, height, T2DM, neck circumference, chest circumference, waist circumference and hip circumference, WHR, WtHR, NHtR), and poorer sleep variables. In addition, patients with OSA had a greater prevalence of hypertension, T2DM and hyperlipidemia, as well as a higher proportion of habitual snoring. Table 1 shows the basic characteristics between the two groups.

\section{Feature Selection}

We used the LASSO logistic regression model to select the most significant predictive features that can help to avoid overfitting. We obtain a more refined model by constructing a penalty function, which makes it compress some regression coefficients. In this study, a linear combination weighted by factor coefficients was used to calculate the risk score, and a cross-validated error plot was produced (Figure 1A). The model with the optimal lambda values included six variables, and the most parsimonious model with a cross-validated error within one standard error of the minimum included three variables. The path of each variable coefficient with varying log-transformed lambda values is displayed in Figure 1B. Therefore, the six features with nonzero coefficients were subsequently selected according to LASSO logistic regression and Clinical importance (gender, habitual snoring, T2DM, neck circumference, BMI, age). In addition, twelve variables (hypertension, hyperlipidemia, hyperuricemia, fatty liver, weight, height, chest circumference, waist circumference and hip circumference, WHR, WtHR, NHtR) with zero coefficients were excluded.

\section{Nomogram Construction}

The six independent predictors (gender, habitual snoring, T2DM, neck circumference, BMI and age) selected using the LASSO logistic regression were included in multivariate logistic regression analysis to establish the nomogram. Table 2 shows the results of the multivariate logistic regression analysis. A nomogram predicting OSA in bariatric surgery candidates was generated. Each predictor corresponds to a specific score by drawing its line in the Points line. The sum of six predictors is located on the total points line, and a vertical line is drawn downward from the total points axis to the risk of OSA axis. Finally, the predicted probability of OSA is obtained in Figure 2.

\section{Validation of the Model}

The nomogram yielded relatively high $\mathrm{C}$-indexes in both the training cohort $(0.856,95 \% \mathrm{CI}: 0.816-0.897)$ and validation cohort (0.829, 95\% CI: 0.76-0.895) which suggested a model with good discrimination. The calibration curve displayed good concordance between prediction and observation in the training and validation cohorts (Figure $3 \mathrm{~A}$ and $\mathrm{B}$ ). The Hosmer-Lemeshow test also showed that there was no significant statistical difference in both training cohorts (Chisquare $=3.942, \quad \mathrm{p}=0.862$ ) and validation cohorts (Chisquare $=6.608, \mathrm{p}=0.374$ ), suggesting the nomogram was well calibrated. In addition, we also evaluated OSA at AHI cutoffs of 5 events/h, 15 events/h and 30 events/h based on the current nomogram using ROC curve analysis. The results showed that the nomogram also has good diagnostic value at AHI cutoffs of 5 events/h $(\mathrm{AUC}=0.856), 15$ events/h (AUC $=0.875)$ and 30 events/h $(\mathrm{AUC}=0.55)$, when AUC of the nomogram is at the optimum cutoff point (Table 3 ).

\section{Clinical Use}

We evaluated the clinical utility of OSA nomogram using the DCA, as is presented in Figure $4 \mathrm{~A}$ and $\mathrm{B}$. The results show that when the nomogram-predicted probability of OSA is $>9 \%$ in training cohorts (Figure $4 \mathrm{~A}$ ) and $>17 \%$ in validation cohort (Figure 4B); this nomogram provides more net benefit than the treat-all-patients scheme or the treat-none scheme. Thus, the nomogram is clinically useful which enables clinicians to make better clinical decisions.

\section{Discussion}

In the present study, the main finding was that the nomogram was accepted as a suitable and easy-to-use tool to screen OSA in patients undergoing evaluation before bariatric surgery. The nomogram was composed of six variables: gender, habitual snoring, T2DM, neck circumference, BMI and age, which were easily available clinically. In fact, although it is simple to use, our nomogram showed adequate discriminatory ability and good predictive performance. Thus, the nomogram assessment tools are promising for bariatric surgery candidates.

There are many risk factors for OSA, including obesity, male gender, age $>50$ years, neck circumference $>40 \mathrm{~cm}$, hypothyroidism, tonsillar or adenoid hypertrophy, craniofacial abnormalities, and lifestyle factors of smoking and alcohol consumption. ${ }^{7,29,30}$ We used LASSO logistic regression to filter out 6 predictors from 18 variables. Three variables (gender, T2DM and $\mathrm{BMI}$ ) with the $\mathrm{P}>$ 
Table I Clinical Characteristics of Patients in the Study

\begin{tabular}{|c|c|c|c|c|c|c|}
\hline \multirow[t]{2}{*}{ Variable } & \multicolumn{2}{|c|}{ Training Cohorts $(n=338)$} & \multirow[t]{2}{*}{$P$ value } & \multicolumn{2}{|c|}{ Validation Cohorts $(n=144)$} & \multirow[t]{2}{*}{$P$ value } \\
\hline & Non-OSA $(n=95)$ & OSA $(n=243)$ & & Non-OSA $(n=45)$ & OSA $(n=99)$ & \\
\hline Gender & & & $<0.001$ & & & 0.001 \\
\hline Male & $13(13.7 \%)$ & $116(47.7 \%)$ & & $9(20.0 \%)$ & $48(48.5 \%)$ & \\
\hline Female & $8286.3 \%)$ & $127(52.3 \%)$ & & $36(80.0 \%)$ & $5 \mathrm{I}(5 \mathrm{I} .5 \%)$ & \\
\hline Habitual snoring & & & $<0.001$ & & & $<0.001$ \\
\hline Yes & $45(47.4 \%)$ & $197(81.1 \%)$ & & $16(35.6 \%)$ & 78(78.8\%) & \\
\hline No & $50(52.6 \%)$ & $46(18.9 \%)$ & & $29(64.4 \%)$ & $21(21.2 \%)$ & \\
\hline Hypertension & & & 0.002 & & & 0.040 \\
\hline Yes & $6(6.3 \%)$ & $49(20.2 \%)$ & & $3(6.7 \%)$ & $20(20.2 \%)$ & \\
\hline No & $89(93.7 \%)$ & 194(79.8\%) & & $42(93.3 \%)$ & $79(79.8 \%)$ & \\
\hline Diabetes mellitus & & & 0.003 & & & 0.023 \\
\hline Yes & $14(14.7 \%)$ & $74(30.5 \%)$ & & $4(8.9 \%)$ & $25(25.3 \%)$ & \\
\hline No & $81(85.3 \%)$ & $169(69.5 \%)$ & & $4 I(91.1 \%)$ & $74(74.7 \%)$ & \\
\hline Dyslipidemia & & & 0.055 & & & 0.070 \\
\hline Yes & $39(41.1 \%)$ & $128(52.7 \%)$ & & $15(33.3 \%)$ & $49(49.5 \%)$ & \\
\hline No & $56(58.9 \%)$ & $115(47.3 \%)$ & & $30(66.7 \%)$ & $50(50.5 \%)$ & \\
\hline Hyperuricemia & & & 0.565 & & & 0.236 \\
\hline Yes & $57(60.0 \%$ & $154(63.4 \%)$ & & $23(51.1 \%)$ & $61(61.6 \%)$ & \\
\hline No & $38(40.0 \%)$ & $89(36.6 \%)$ & & $22(48.9 \%)$ & $38(38.4 \%)$ & \\
\hline Fatty liver & & & 0.278 & & & 0.694 \\
\hline Yes & $69(72.6 \%)$ & $190(78.2 \%)$ & & $31(68.9 \%)$ & $72(72.7 \%)$ & \\
\hline No & $26(27.4 \%)$ & $53(21.8 \%)$ & & $14(3 \mid .1 \%)$ & $27(27.3 \%)$ & \\
\hline Age (years) & $25.9 \pm 8.3$ & $32.6 \pm 10.1$ & 0.000 & $26.5 \pm 8.4$ & $31.6 \pm 11.5$ & 0.009 \\
\hline Height (cm) & $163.5 \pm 11.5$ & $167.4 \pm 9.9$ & 0.002 & $165.3 \pm 7.5$ & $166.1 \pm 11.0$ & 0.639 \\
\hline Weight(kg) & $99.6 \pm 23.0$ & $114.7 \pm 27.8$ & 0.000 & $96.5 \pm 18.9$ & $114.3 \pm 26.9$ & 0.000 \\
\hline BMI (kg/m2) & $35.9 \pm 5.6$ & $40.7 \pm 8.4$ & 0.000 & $35.0 \pm 5.1$ & $40.8 \pm 8.5$ & 0.000 \\
\hline $\mathrm{NC}(\mathrm{cm})$ & $39.0 \pm 3.5$ & $43.5 \pm 5.0$ & 0.000 & $39.9 \pm 4.1$ & $43.5 \pm 5.0$ & 0.000 \\
\hline $\mathrm{CC}(\mathrm{cm})$ & $113.5 \pm 13.0$ & $125.3 \pm 16.5$ & 0.000 & $114.5 \pm 9.5$ & $123.5 \pm \mid 4.1$ & 0.000 \\
\hline WC $(\mathrm{cm})$ & $114.9 \pm 10.4$ & $|24.1 \pm| 4.1$ & 0.000 & $113.0 \pm 11.4$ & $125.2 \pm 17.6$ & 0.000 \\
\hline $\mathrm{HC}(\mathrm{cm})$ & $119.2 \pm 11.3$ & $126.7 \pm 15.0$ & 0.000 & $118.4 \pm 10.9$ & $26.7 \pm 15.6$ & 0.000 \\
\hline WHR & $0.95 \pm 0.07$ & $0.99 \pm 0.07$ & 0.000 & $0.96 \pm 0.05$ & $0.99 \pm 0.08$ & 0.002 \\
\hline WtHR & $0.24(0.23,0.25)$ & $0.26(0.24,0.28)$ & 0.000 & $0.24(0.22,0.25)$ & $0.26(0.25,0.28)$ & 0.000 \\
\hline NtHR & $0.68(0.64,0.73)$ & $0.74(0.68,0.80)$ & 0.000 & $0.68(0.64,0.74)$ & $0.74(0.69,0.81)$ & 0.000 \\
\hline Total sleep time (min) & $428.0 \pm 84.8$ & $393.8 \pm 90.2$ & 0.002 & $448.8 \pm 77.5$ & $387.7 \pm 93.4$ & 0.000 \\
\hline Sleep efficiency (\%) & $89.3(78,94.3)$ & $77.0(63.0,88.7)$ & 0.000 & $90.3(80.2,93.8($ & $77.6(60.8,88.1)$ & 0.000 \\
\hline $\mathrm{AHI}$ & $1.1(0.3,2.5)$ & $23.6(\mid 2.4,54.9)$ & 0.000 & $1.2(0.4,2.7)$ & $22.6(\mid 0.1,51.6)$ & 0.000 \\
\hline Arousal index & $6.7(5.1,9.6)$ & $23.9(\mid 2.4,39.3)$ & 0.000 & $6.6(5.2,10.6)$ & $22.2(9.4,40.1)$ & 0.000 \\
\hline Mean $\mathrm{SaO} 2$ (\%) & $97.0(96.0,97.0)$ & $94.0(92.0,95.0)$ & 0.000 & $96.0(96.0,97.0)$ & $95.0(93.0,96.0)$ & 0.000 \\
\hline Minimum $\mathrm{SaO} 2$ (\%) & $90.0(87.0,92.0)$ & $79.0(65.0,85.0)$ & 0.000 & $90.0(86.0,91.0)$ & $80.0(70.0,87.0)$ & 0.000 \\
\hline
\end{tabular}

Abbreviations: BMI, body mass index; NC, neck circumference; CC, chest circumference; WC, waist circumference; HC, hip circumference; WHR, waist to hip ratio; WHtR, waist to height ratio; $\mathrm{NHtR}$, neck to height ratio; $\mathrm{AHI}$, apnoea-hypopnea index; $\mathrm{SaO}$, oxygen saturation. 

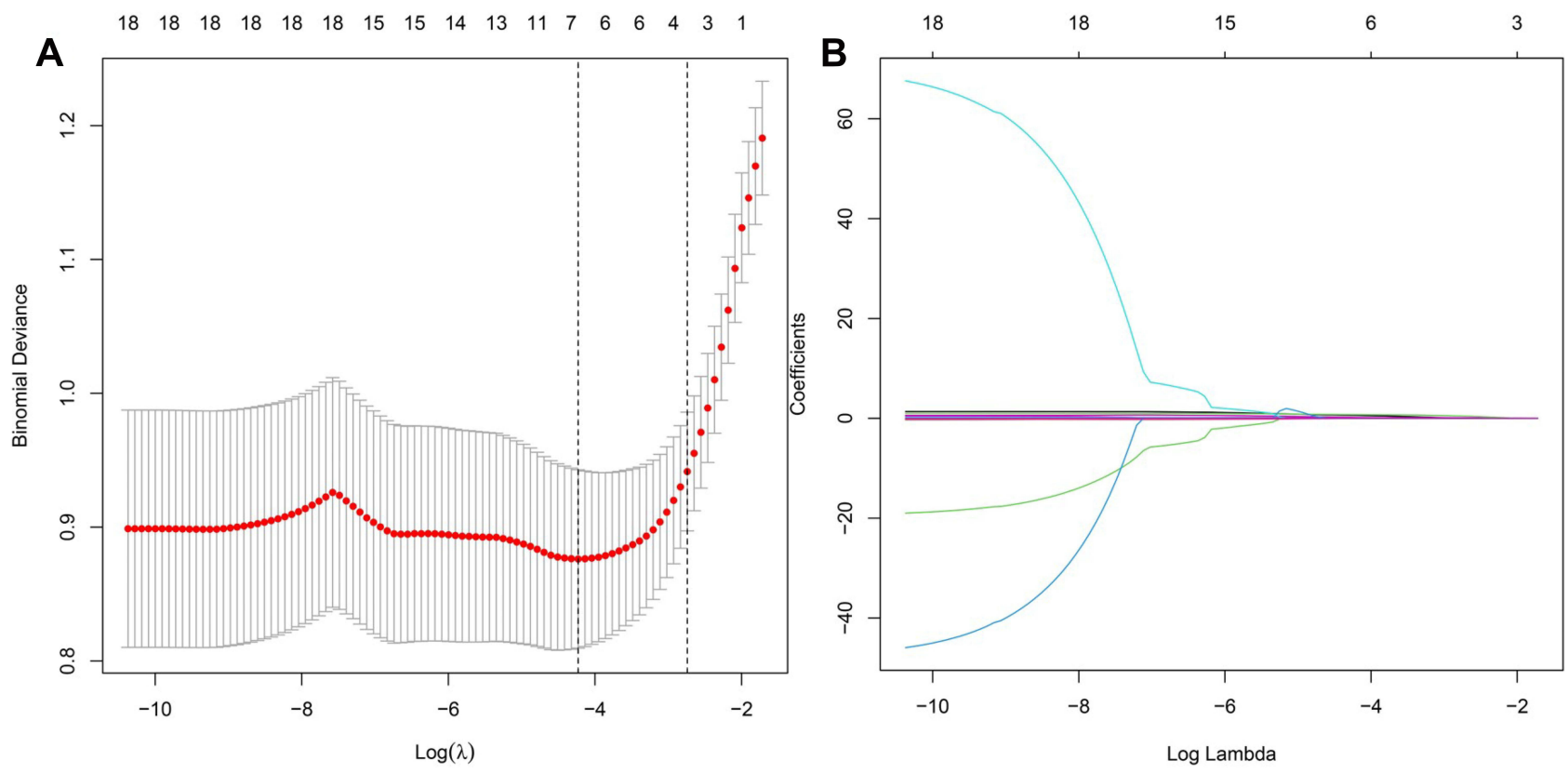

Figure I Clinical feature selection using the least absolute shrinkage and selection operator (LASSO) logistic regression. (A). Optimal parameter (lambda) selection in the LASSO logistic regression used 10 -fold cross-validation via minimum criteria. The black vertical lines were drawn at the optimal values by using the minimum criteria and the one standard error of the minimum criteria (the I-SE criteria). (B) LASSO coefficient profiles of the 18 features. A coefficient profile plot was produced against the log (lambda) sequence.

0.05 were included in the model, due to considering variables closely associated with OSA. Therefore, the nomograph included 5 objective parameters (gender, T2DM, neck circumference, BMI and age) and 1 subjective parameter (habitual snoring). Of those variables, gender, age, neck circumference and BMI are well-known predictors of OSA. Habitual snoring is a common symptom in patients with OSA, and many questionnaires or models regard it as an important predictor. $^{31,32}$ There is sufficient evidence that T2DM is closely related to OSA. ${ }^{33,34}$ Shi et al ${ }^{35}$ established a nomogram that incorporated 6 independent factors (WHR, smoking status, BMI, uric acid, homeostasis model assessment insulin resistance index, and fatty liver) to predict OSA in patients with T2DM. Thus, these predictors are reasonable for the construction of the model.
Validation of the nomogram is important to avoid overfitting and to determine generalizability. ${ }^{23}$ In the current study, the nomogram showed favorable discrimination in both the training cohort $(\mathrm{C}$-index $=0.856)$ and validation cohort $(\mathrm{C}$-index $=0.829$ ). Furthermore, calibration curves showed optimal agreement between the predicted probabilities and observed outcomes. DCA also indicated that if the threshold probability of a patient was $>9 \%$ (training cohort) and $>17 \%$ (validation cohort), using this nomogram added more benefit than the treat-all-patients scheme or the treat-none scheme, which suggested that the nomogram was clinically useful. In addition, the nomogram also provided adequate accuracy for OSA screening at $A H I \geq 15 / h$ or $A H I \geq 30 / h$ (Table 3 ). Therefore, our nomogram was proved to be robust.

Table 2 Prediction Factors for Obstructive Sleep Apnea in Bariatric Surgery Candidates

\begin{tabular}{|l|l|l|l|l|l|}
\hline Intercept and Variable & $\boldsymbol{\beta}$ & $\mathbf{9 5 \%} \mathbf{C l}$ & $\mathbf{O R}$ & $\mathbf{9 5 \%} \mathbf{C I}$ & $\mathbf{P}$ \\
\hline Intercept & -11.476 & $(-16.021,-7.389)$ & $<0.001$ & - & 0.000 \\
Gender & 0.887 & $(-0.026,1.829)$ & 2.429 & $(0.974,6.230)$ & 0.060 \\
Habitual snoring & 0.826 & $(0.169,1.487)$ & 2.283 & $(1.184,4.425)$ & 0.014 \\
Diabetes mellitus & 0.436 & $(-0.313,1.226)$ & 1.547 & $(0.731,3.409)$ & 0.264 \\
Neck circumference & 0.160 & $(0.045,0.281)$ & 1.173 & $(1.046,1.325)$ & 0.008 \\
BMI & 0.050 & $(-0.013,0.118)$ & 1.052 & $(0.987, I .125)$ & 0.130 \\
Age & 0.107 & $(0.071,0.147)$ & 1.113 & $(1.073,1.159)$ & 0.000 \\
\hline
\end{tabular}

Abbreviations: $\beta$, the regression coefficient; OR, odds ratio; $\mathrm{Cl}$, confidence interval; $\mathrm{BMI}$, body mass index. 
Points

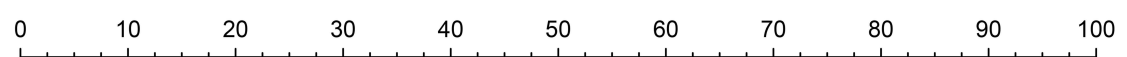

Gender

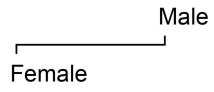

Habitual snoring

Yes

No

T2DM

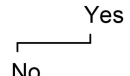

Neck circumference

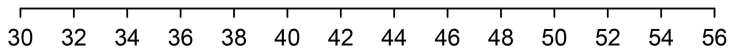

$\mathrm{BMI}$

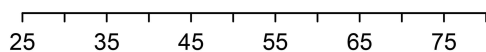

Age

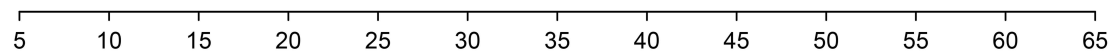

Total Points

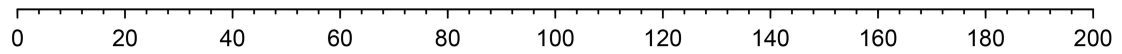

Risk of OSA

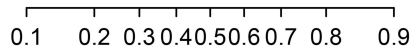

Figure 2 Nomogram to predict OSA in bariatric surgery candidates.

Abbreviations: OSA, obstructive sleep apnea; T2DM, type 2 diabetes mellitus; BMI, body mass index.
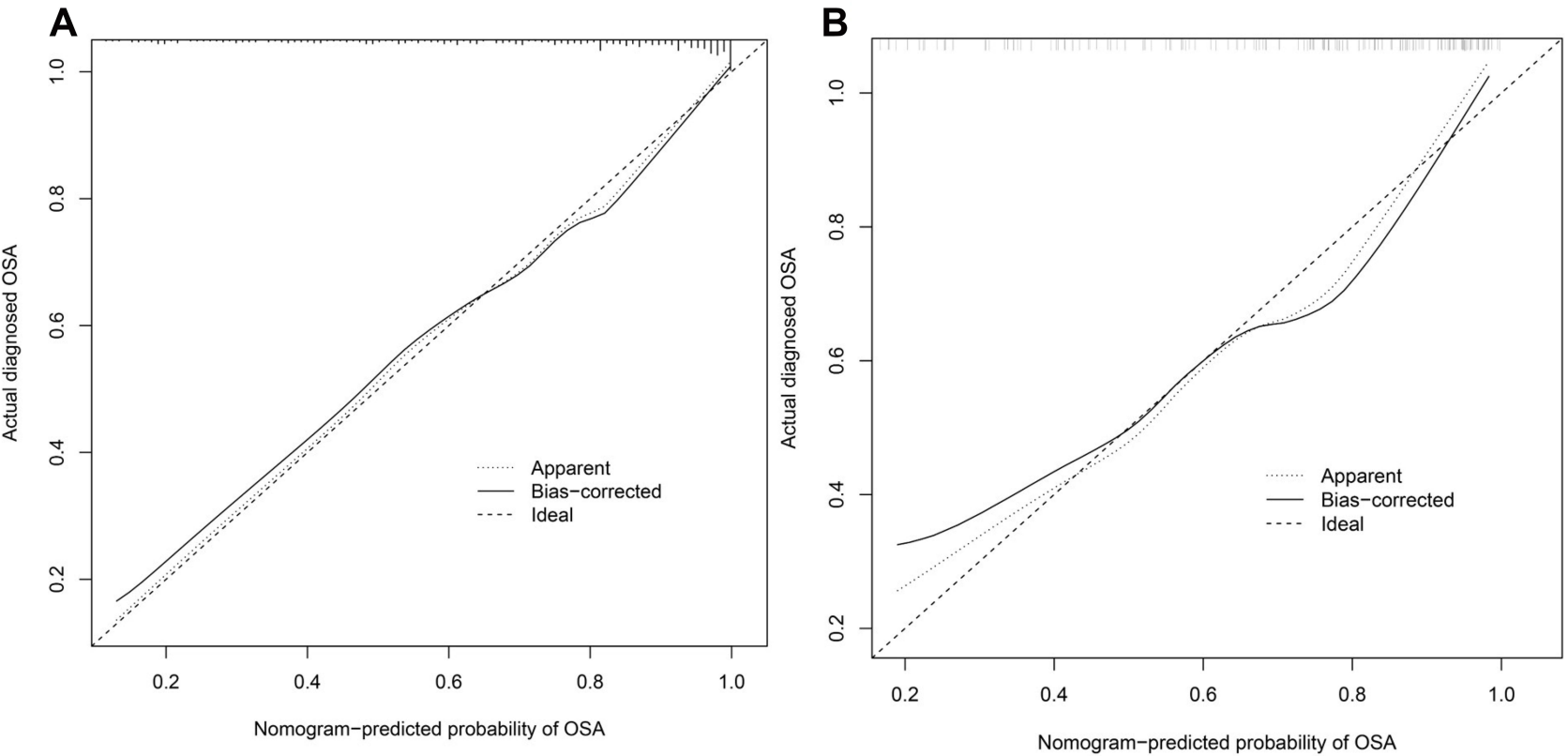

Figure 3 Calibration curves for the nomogram in the training cohort $(\mathbf{A})$ and validation cohort $(\mathbf{B})$. The $x$-axis represents the nomogram-predicted OSA risk. The $y$-axis is the actual diagnosed OSA. The dotted line represents a perfect prediction by an ideal model. The solid line represents the performance of the nomogram, of which a closer fit to the diagonal dotted line represents a better prediction. 
Table 3 The Clinical Utility of the Nomogram for Detecting Obstructive Sleep Apnea

\begin{tabular}{|l|l|l|l|l|l|l|l|}
\hline AHI & AUC (95\% CI) & Sensitivity (95\% CI) & Specificity (95\% CI) & \multicolumn{2}{|l|}{ Predictive Value (95\% CI) } & \multicolumn{2}{l|}{ Likelihood Ratio (95\% CI) } \\
\cline { 5 - 8 } & & & & Positive & Negative & Positive & Negative \\
\hline$\geq 5$ & $0.856(0.814-0.892)$ & $68.72(62.5-74.5)$ & $87.37(79.0-93.3)$ & $93.3(88.6-96.5)$ & $52.2(44.1-60.2)$ & $5.44(4.9-6.1)$ & $0.36(0.2-0.6)$ \\
$\geq 15$ & $0.875(0.834-0.908)$ & $79.62(72.5-85.6)$ & $82.32(76.0-87.6)$ & $79.6(72.5-85.6)$ & $82.3(75.9-87.6)$ & $4.50(4.1-5.0)$ & $0.25(0.2-0.4)$ \\
$\geq 30$ & $0.855(0.813-0.891)$ & $89.11(81.3-94.4)$ & $69.20(62.9-75.0)$ & $55.2(47.2-63.0)$ & $93.7(89.0-96.8)$ & $2.89(2.6-3.2)$ & $0.16(0.09-0.3)$ \\
\hline
\end{tabular}

Abbreviations: AHI, apnoea-hypopnea index; AUC, area under the curve.

With the ever-increasing prevalence of obesity worldwide, an increase in bariatric surgery in clinical practice has been seen. ${ }^{36}$ Therefore, it would be important to find a feasible tool to detect OSA preoperatively due to the high prevalence and potentially perioperative risk of OSA. Screening tools such as the Berlin questionnaire, ESS, STOP-Bang and STOP questionnaire (STOP) have been widely used for detecting OSA. ${ }^{16}$ However, the optimal tool for the preoperative evaluation of OSA in this specific population is unclear. In a study that included 814 bariatric surgery patients, GOAL, No-Apnea, STOP-Bang, and NoSAS, but not ESS and STOP, enabled satisfactory discrimination as OSA screening tools, but the accuracy of these screening tools is less than 0.8 (AUC $<0.8$ ). ${ }^{17}$ Nonetheless, a retrospective study of 266 patients who underwent bariatric surgery, the results showed that neither STOP-Bang nor Berlin questionnaires were suitable as screening tools for OSA. ${ }^{37}$ A prediction model including 13 variables for
OSA created by Kolotkin et al was used in bariatric surgery patients, but it was very complicated to use and did have inadequate sensitivity and specificity. ${ }^{38} \mathrm{Xu}$ et al developed and validated a nomogram including 8 variables (age, sex, neck circumference, apolipoprotein B, glucose, insulin, BMI and waist circumference) and showed good accuracy and discrimination, ${ }^{39}$ but it included some biochemical parameters which made the use of this nomogram more complicated. In the present study, the nomogram performed well and served as an effective screening tool to identify patients with OSA before bariatric surgery because both training and validation cohorts had high accuracy (C-index $>0.8)$. It is worth noting that the primary purpose of the nomogram is to help bariatric surgeons to select those who emerge as high-risk OSA to PSG, but not to replace the standard diagnostic method. More importantly, depending on the nomogram, bariatric surgeons could address such screening issues that the
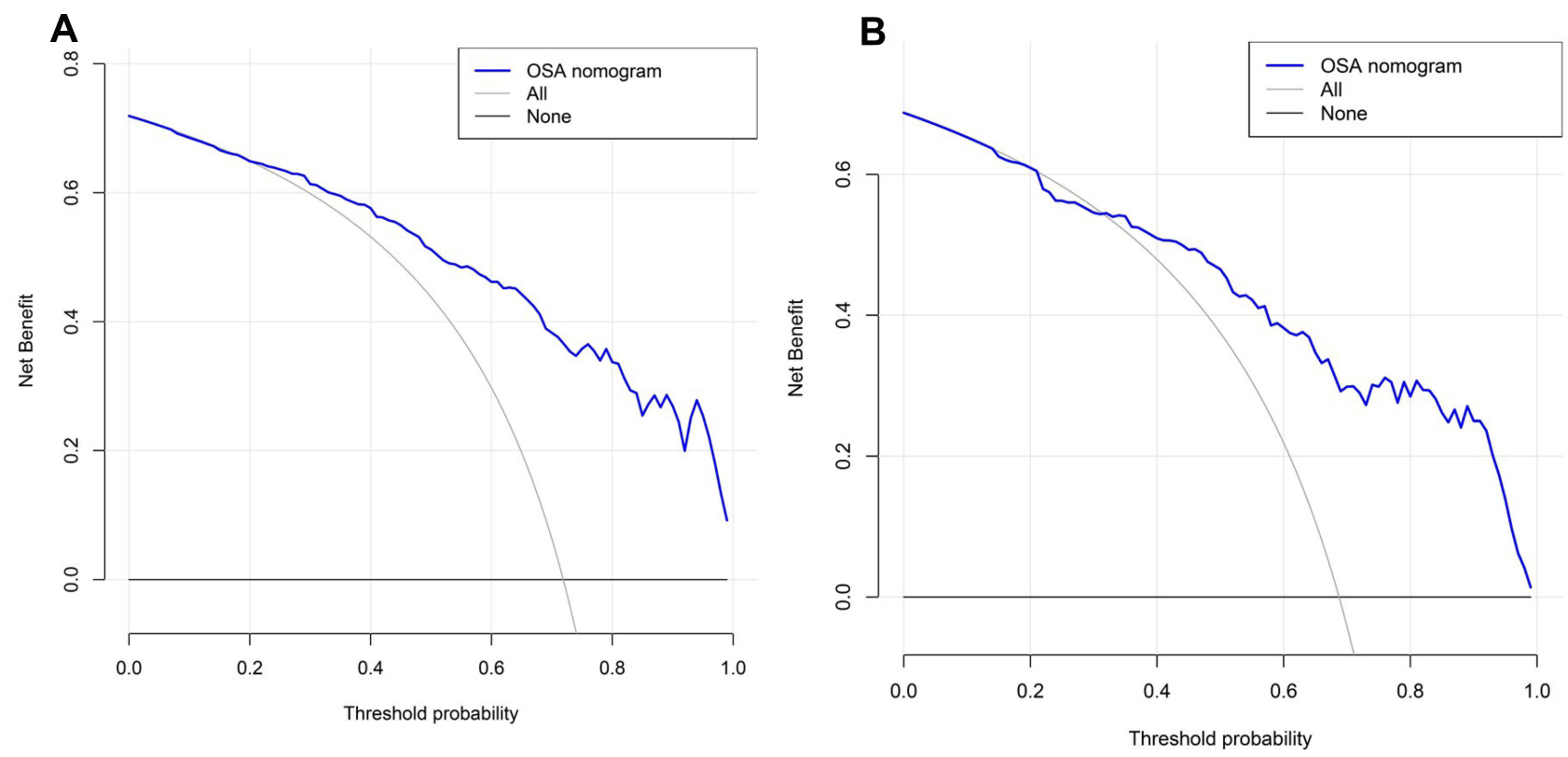

Figure 4 Decision curve analysis for the OSA nomogram in the training cohort $(\mathbf{A})$ and validation cohort (B). The $y$-axis measures the net benefit. The blue line represents the nomogram. The black line represents the assumption of intervention-none. The gray line represents the assumption of intervention-all-patients. The decision curve showed that if the threshold probability of a patient and a doctor is $>9 \%$ (training cohorts) and $>17 \%$ (validation cohorts), respectively, using this nomogram to predict OSA risk adds more benefit than the treat-all-patients scheme or the treat-none scheme. 
patient refuses to undergo PSG but has a high-risk OSA, thereby reducing the use of costly and time-consuming PSG.

The ultimate purpose of using nomograms is to help clinicians and patients to interpret individual needs for additional intervention or treatment. In this study, in order to prove its clinical usefulness, decision curve analysis was used to evaluate whether decision-making using OSA nomograms would improve patient outcomes. The results showed that the threshold probability of a patient was $>9 \%$ in training cohort, which meant that all patients can benefit from using this model, but the degree of benefit was different. For example, if the patient would opt for a sleep study if his probability of OSA was $60 \%$, then the net benefit is 0.44 , when using the nomogram to make the decision of whether to undergo intervention, with added benefit than the treat-all scheme or the treat-none scheme. Therefore, those patients with a high pretest for OSA should decide to undergo PSG monitoring as soon as possible. Generally speaking, these patients with moderate OSA (AHI 15.0-29.9) and symptomatic or severe OSA (AHI $\geq 30$ ) should be recommended continuous positive airway pressure (CPAP). ${ }^{40}$ Our model may be difficult to distinguish whether the patient is at risk of moderate or severe OSA. There is still a lack of evidence for decisionmaking in CAPA treatment through this model. In future research, we can further explore whether to perform CAPA treatment by establishing a model.

This study has several limitations. First, our nomogram was based on a retrospective data collection from a single center and we are unable to collect data regarding ESS and STOP-Bang, which may compromise the generalization of our results. Secondly, there was a lack of external data to validate the model, so whether these findings can be widely used in other populations is unclear. Thirdly, some factors that may be associated with OSA were not considered, such as smoking status, cardiovascular disease and racial differences. Given these limitations, further efforts on prospective and multicenter data collection are encouraged to prove the robustness of nomogram. However, our nomogram can provide a screening tool for bariatric surgeons to evaluate the risk of OSA in bariatric surgery candidates, which is more important for those who refuse to undergo PSG before bariatric surgery. In addition, this novel nomogram helps obese patients to assess the risk of OSA and better make decisions to further refer them to a sleep center for diagnostic PSG.

\section{Conclusions}

In conclusion, we developed and validated a novel nomogram with adequate performance and discrimination for predicting OSA in bariatric surgery candidates, regardless of the severity level. The nomogram could serve as a concise and practical tool to help clinicians select bariatric surgery patients with high-risk OSA to PSG. Despite the promising prospect, further prospective and multicenter studies are warranted to validate the robustness of the proposed nomogram.

\section{Funding}

This research was supported by the First Affiliated Hospital of Jinan University Flagship Specialty Construction Project-General Surgery (No.711003).

\section{Disclosure}

The authors declare no conflicts of interest for this work.

\section{References}

1. Kapur VK, Auckley DH, Chowdhuri S, et al. Clinical practice guideline for diagnostic testing for adult obstructive sleep apnea: an American Academy of Sleep Medicine Clinical Practice Guideline. $J$ Clin Sleep Med. 2017;13(3):479-504. doi:10.5664/jcsm.6506

2. Gottlieb DJ, Punjabi NM. Diagnosis and management of obstructive sleep apnea: a review. JAMA. 2020;323(14):1389-1400. doi:10.1001/ jama.2020.3514

3. Gaines J, Vgontzas AN, Fernandez-Mendoza J, Bixler EO. Obstructive sleep apnea and the metabolic syndrome: the road to clinically-meaningful phenotyping, improved prognosis, and personalized treatment. Sleep Med Rev. 2018;42:211-219. doi:10.1016/j.smrv.2018.08.009

4. Javaheri S, Barbe F, Campos-Rodriguez F, et al. Sleep apnea: types, mechanisms, and clinical cardiovascular consequences. J Am Coll Cardiol. 2017;69(7):841-858. doi:10.1016/j.jacc.2016.11.069

5. Somers VK, White DP, Amin R, et al. Sleep apnea and cardiovascular disease: an American Heart Association/American College Of Cardiology Foundation Scientific Statement from the American Heart Association Council for High Blood Pressure Research Professional Education Committee, Council on Clinical Cardiology, Stroke Council, and Council On Cardiovascular Nursing. In collaboration with the National Heart, Lung, and Blood Institute National Center on Sleep Disorders Research (National Institutes of Health). Circulation. 2008;118(10):1080-1111. doi:10.1161/ CIRCULATIONAHA.107.189375

6. Peppard PE, Young T, Palta M, Dempsey J, Skatrud J. Longitudinal study of moderate weight change and sleep-disordered breathing. JAMA. 2000;284(23):3015-3021. doi:10.1001/jama.284.23.3015

7. Young T, Skatrud J, Peppard PE. Risk factors for obstructive sleep apnea in adults. JAMA. 2004;291(16):2013-2016. doi:10.1001/ jama.291.16.2013

8. Peromaa-Haavisto P, Tuomilehto H, Kössi J, et al. Prevalence of obstructive sleep apnoea among patients admitted for bariatric surgery. A prospective multicentre trial. Obes Surg. 2016;26(7):1384-1390.

9. Arterburn D, Wellman R, Emiliano A, et al. Comparative effectiveness and safety of bariatric procedures for weight loss: a PCORnet Cohort Study. Ann Intern Med. 2018;169(11):741-750. doi:10.7326/M172786 
10. Loo GH, Rajan R, Mohd Tamil A, Ritza Kosai N. Prevalence of obstructive sleep apnea in an Asian bariatric population: an underdiagnosed dilemma. Surg Obes Relat Dis. 2020;16(6):778-783. doi:10.1016/j.soard.2020.02.003

11. Rasmussen JJ, Fuller WD, Ali MR. Sleep apnea syndrome is significantly underdiagnosed in bariatric surgical patients. Surg Obes Relat Dis. 2012;8(5):569-573. doi:10.1016/j.soard.2011.06.021

12. Opperer M, Cozowicz C, Bugada D, et al. Does obstructive sleep apnea influence perioperative outcome? A qualitative systematic review for the society of anesthesia and sleep medicine task force on preoperative preparation of patients with sleep-disordered breathing. Anesth Analg. 2016;122(5):1321-1334. doi:10.1213/ ANE.0000000000001178

13. Kong WT, Chopra S, Kopf M, et al. Perioperative risks of untreated obstructive sleep apnea in the bariatric surgery patient: a Retrospective Study. Obes Surg. 2016;26(12):2886-2890. doi:10.1007/s11695-016-2203-3

14. Epstein LJ, Kristo D, Strollo PJ Jr, et al. Clinical guideline for the evaluation, management and long-term care of obstructive sleep apnea in adults. J Clin Sleep Med. 2009;5(3):263-276.

15. Mirrakhimov AE, Sooronbaev T, Mirrakhimov EM. Prevalence of obstructive sleep apnea in Asian adults: a systematic review of the literature. BMC Pulm Med. 2013;13:10. doi:10.1186/1471-2466-1310

16. Chiu HY, Chen PY, Chuang LP, et al. Diagnostic accuracy of the Berlin questionnaire, STOP-BANG, STOP, and Epworth sleepiness scale in detecting obstructive sleep apnea: a bivariate meta-analysis. Sleep Med Rev. 2017;36:57-70. doi:10.1016/j.smrv.2016.10.004

17. Duarte RLM, Magalhães-da-silveira FJ, Gozal D. Validation of the GOAL questionnaire as an obstructive sleep apnea screening instrument in bariatric surgery candidates: a Brazilian Single-Center Study. Obes Surg. 2020;30(12):4802-4809. doi:10.1007/s11695-020-048884

18. Duarte RLM, Mello FCQ, Magalhães-da-silveira FJ, et al. Comparative performance of screening instruments for obstructive sleep apnea in morbidly obese patients referred to a sleep laboratory: a prospective cross-sectional study. Sleep Breath. 2019;23(4):11231132. doi:10.1007/s11325-019-01791-w

19. Vasas P, Gupta A, Owers C, et al. Obstructive sleep apnoea screening preoperatively with the Epworth questionnaire: is it worth it ... ? Obes Surg. 2019;29(3):851-857. doi:10.1007/s11695-018-3600-6

20. Horvath CM, Jossen J, Kröll D, et al. Prevalence and prediction of obstructive sleep apnea prior to bariatric surgery-gender-specific performance of four sleep questionnaires. Obes Surg. 2018;28(9):27202726. doi:10.1007/s11695-018-3222-z

21. Duarte RLDM, Magalhães-da-silveira FJ. Factors predictive of obstructive sleep apnea in patients undergoing pre-operative evaluation for bariatric surgery and referred to a sleep laboratory for polysomnography. J Bras Pneumol. 2015;41(5):440-448. doi:10.1590/ S1806-37132015000000027

22. Park SY. Nomogram: an analogue tool to deliver digital knowledge. $J$ Thorac Cardiovasc Surg. 2018;155(4):1793. doi:10.1016/j. jtcvs.2017.12.107

23. Iasonos A, Schrag D, Raj GV, Panageas KS. How to build and interpret a nomogram for cancer prognosis. J Clin Oncol. 2008;26 (8):1364-1370. doi:10.1200/JCO.2007.12.9791

24. Kasama K, Mui W, Lee WJ, et al. IFSO-APC consensus statements 2011. Obes Surg. 2012;22(5):677-684. doi:10.1007/s11695-0120610-7
25. Teculescu D, Benamghar L, Hannhart B, Michaely JP. Habitual loud snoring. A study of prevalence and associations in 850 middle-aged French males. Respiration. 2006;73(1):68-72. doi:10.1159/ 000088355

26. Berry RB, Budhiraja R, Gottlieb DJ, et al. Rules for scoring respiratory events in sleep: update of the 2007 AASM manual for the scoring of sleep and associated events. Deliberations of the sleep apnea definitions task force of the American Academy of Sleep Medicine. J Clin Sleep Med. 2012;8(5):597-619. doi:10.5664/ jcsm. 2172

27. Sauerbrei W, Royston P, Binder H. Selection of important variables and determination of functional form for continuous predictors in multivariable model building. Stat Med. 2007;26(30):5512-5528. doi: $10.1002 / \operatorname{sim} .3148$

28. Vickers AJ, Cronin AM, Elkin EB, Gonen M. Extensions to decision curve analysis, a novel method for evaluating diagnostic tests, prediction models and molecular markers. BMC Med Inform Decis Mak. 2008;8:53. doi:10.1186/1472-6947-8-53

29. Wilhelm CP, deShazo RD, Tamanna S, Ullah MI, Skipworth LB. The nose, upper airway, and obstructive sleep apnea. Ann Allergy Asthma Immunol. 2015;115(2):96-102. doi:10.1016/j.anai.2015.06.011

30. Tan HL, Kheirandish-Gozal L, Abel F, Gozal D. Craniofacial syndromes and sleep-related breathing disorders. Sleep Med Rev. 2016;27:74-88. doi:10.1016/j.smrv.2015.05.010

31. Kreitinger KY, Lui MMS, Owens RL, et al. Screening for obstructive sleep apnea in a diverse bariatric surgery population. Obesity. 2020;28(11):2028-2034. doi:10.1002/oby.23021

32. Luo M, Zheng HY, Zhang Y, et al. A nomogram for predicting the likelihood of obstructive sleep apnea to reduce the unnecessary polysomnography examinations. Chin Med J. 2015;128(16):21342140. doi:10.4103/0366-6999.162514

33. Wang X, Bi Y, Zhang Q, Pan F. Obstructive sleep apnoea and the risk of type 2 diabetes: a meta-analysis of prospective cohort studies. Respirology. 2013;18(1):140-146. doi:10.1111/j.14401843.2012.02267.x

34. Rajan P, Greenberg H. Obstructive sleep apnea as a risk factor for type 2 diabetes mellitus. Nat Sci Sleep. 2015;7:113-125. doi:10.2147/ NSS.S90835

35. Shi H, Xiang S, Huang X, et al. Development and validation of a nomogram for predicting the risk of obstructive sleep apnea in patients with type 2 diabetes. Ann Transl Med. 2020;8(24):1675. doi: $10.21037 / \mathrm{atm}-20-6890$

36. Angrisani L, Santonicola A, Iovino P, et al. Bariatric surgery and endoluminal procedures: IFSO worldwide survey 2014. Obes Surg. 2017;27(9):2279-2289. doi:10.1007/s11695-017-2666-x

37. Glazer SA, Erickson AL, Crosby RD, et al. The evaluation of screening questionnaires for obstructive sleep apnea to identify high-risk obese patients undergoing bariatric surgery. Obes Surg. 2018;28 (11):3544-3552. doi:10.1007/s11695-018-3391-9

38. Kolotkin RL, LaMonte MJ, Walker JM, et al. Predicting sleep apnea in bariatric surgery patients. Surg Obes Relat Dis. 2011;7(5):605610. doi:10.1016/j.soard.2011.04.226

39. Xu H, Zhao X, Shi Y, et al. Development and validation of a simpleto-use clinical nomogram for predicting obstructive sleep apnea. BMC Pulm Med. 2019;19(1):18. doi:10.1186/s12890-019-0782-1

40. Mingrone G, Bornstein S, Le Roux CW. Optimisation of follow-up after metabolic surgery. Lancet Diabetes Endocrinol. 2018;6(6):487499. doi:10.1016/S2213-8587(17)30434-5 


\section{Publish your work in this journal}

Nature and Science of Sleep is an international, peer-reviewed, open access journal covering all aspects of sleep science and sleep medicine, including the neurophysiology and functions of sleep, the genetics of sleep, sleep and society, biological rhythms, dreaming, sleep disorders and therapy, and strategies to optimize healthy sleep.
The manuscript management system is completely online and includes a very quick and fair peer-review system, which is all easy to use. Visit http://www.dovepress.com/testimonials.php to read real quotes from published authors. 